\title{
Existe preparação dos músculos do assoalho pélvico na assistência pré-natal? Uma avaliação de mulheres acompanhadas em uma unidade básica de saúde
}

\author{
Is there preparation of the pelvic floor muscles in prenatal care? \\ An evaluation of women followed at a basic health unit.
}

ANDRESSA CÂMARa Rodrigues ${ }^{1}$ VANessa ÂNGela de Oliveira ${ }^{1}$ SaÚl Rassy Carneiro Cibele Nazaré CÂmara RODRIGUES ${ }^{\text {II }}$

Dayse Danielle De O. Silva ${ }^{I I I}$ Gustavo F. Sutter Latorre Erica Feio Carneiro Nunes ${ }^{\text {III }}$ ${ }^{1}$ Universidade da AMAZÔNIA, BELÉM/PA - BRASIL

"I Universidade Federal do Pará, BELÉM/PA - BRASIL

III UNIVERSIDADE DO ESTADO DO Pará, Belém/PA - Brasil
Resumo Introdução: A fisioterapia como forma de tratamento dos músculos do assoalho pélvico (MAP) na gravidez é uma alternativa eficaz na redução dos índices de disfunções do assoalho pélvico. No entanto, no Brasil ainda são poucas as mulheres gestantes que são informadas a respeito da necessidade de realizar uma avaliação do assoalho pélvico e da importância de exercitá-lo.

Objetivo: Observar se a puérpera recebeu orientação durante o pré-natal para exercitar os músculos do assoalho pélvico. Metodologia: Foi utilizado o Questionário de Experiência e Satisfação com o Parto modificado para a coleta de dados com mulheres entre 18 a 40 anos de idade no Centro Saúde Escola do Marco, localizado na cidade de Belém-PA, no período de janeiro a abril de 2017.

Resultados: Participaram do estudo, 199 puérperas. O parto vaginal foi o mais incentivado, realizado e desejado. Das entrevistadas, $60,80 \%$ não conheciam os músculos do assoalho pélvico e não receberam orientação para a preparação dos mesmos. Não apresentaram perda de urina, $61,31 \%$, e $85,43 \%$ não tiveram perda de fezes.

Conclusão: A maioria das mulheres não recebeu orientação para preparar os MAP para o parto durante o pré-natal e apenas a minoria tinha conhecimento sobre o que é o assoalho pélvico.

Palavras-chave: Gravidez. Fisioterapia. Assoalho pélvico. Pré-NATAL.

ABSTRACT Introduction: Physiotherapy as a way of treating pelvic floor muscles (PFM) in pregnancy is an effective alternative in reducing pelvic floor dysfunction rates. However, in Brazil there are still few pregnant women who are informed about the need to carry out an assessment of the pelvic floor and the importance of exercising them.

Objective: Observe if the puerperal woman received guidance during prenatal care to exercise the pelvic floor muscles. Methodology: The modified Childbirth Experience and Satisfaction Questionnaire was used to collect data with women between 18 and 40 years old at Centro Saúde Escola do Marco, located in the city of Belém-PA, from January to April 2017. Results: Participated in the study 199 puerperal women. Vaginal delivery was the most encouraged, performed and desired. Of the interviewees, $60.80 \%$ did not know the pelvic floor muscles and did 
not receive guidance on how to prepare them. $61.31 \%$ did not present loss of urine and $85.43 \%$ did not present loss of feces.

Conclusion: Most women were not instructed to prepare PFM for delivery during prenatal care and only a minority were aware of what the pelvic floor is.

Keywords: Pregnancy. Pheromones. Pelvic floor. Prenatal care.

\section{INTRODUÇÃO}

Engravidar é o sonho de muitos casais e para ter uma gravidez saudável é necessário que o pré-natal ocorra adequadamente, possibilitando que a gestação, o parto e o puerpério sejam uma experiência humana benéfica e enriquecedora. ${ }^{1}$

O corpo da gestante se transforma gradualmente para acomodar o feto durante suas fases de desenvolvimento, ao mesmo tempo em que se prepara para o parto. Entretanto, algumas alterações que acontecem no corpo da gestante, como o aumento de peso, podem resultar em sobrecarga nos músculos do assoalho pélvico (MAP)., ${ }^{2,3}$

Os MAP fazem parte de um conjunto de partes moles responsáveis por sustentar os órgãos pélvicos e abdominais denominados assoalho pélvico. ${ }^{4}$ As alterações decorrentes da gravidez e parto vaginal podem favorecer o surgimento das chamadas disfunções do assoalho pélvico (DAP), representadas pela incontinência urinária (IU), incontinência anal, prolapso dos órgãos pélvicos e disfunção sexual. ${ }^{5}$

$\mathrm{O}$ assoalho pélvico tem papel importante nas funções urinária, anal, reprodutiva e sexual. Contudo, não existe serviços, no Brasil, que informam acerca da necessidade da avaliação do assoalho pélvico, bem como, dos benefícios do treinamento dos MAP para o parto vaginal. ${ }^{6}$

Estima-se que 21,7\% das mulheres, no Brasil, entre 18 a 45 anos de idade apresen- tam algum tipo de $\mathrm{DAP}^{7}$ e que $28 \%$ a $64 \%$ das mulheres desenvolvem IU durante a gravidez ${ }^{8}$. Entretanto, os dados da prevalência não são exatos, visto que uma parcela da população não relata os seus sintomas. ${ }^{9}$

Existe uma forte tendência de que essas disfunções se tornem cada vez mais frequentes, que atualmente a IU é considerada um problema de saúde pública ${ }^{10}$ que afeta cerca $22,9 \%$ das jovens nulíparas ${ }^{11}$ e pode atingir $40 \%$ das mulheres com idade entre 60 e 91 anos. ${ }^{12}$ A fisioterapia, como forma de tratamento dos MAP na gravidez, se mostra uma alternativa eficaz na redução dos índices de DAP. ${ }^{13}$

Considerando a importância de um protocolo fisioterapêutico durante o pré-natal voltado para a preparação dos MAP, o presente estudo teve como objetivo observar se as puérperas receberam orientação durante o pré-natal para exercitar os MAP e a sua satisfação quanto ao pré-natal recebido.

\section{Metodologia}

Trata-se de um estudo observacional transversal, aprovado pelo Comitê de Ética em Pesquisa da Universidade do Estado do Pará (UEPA), com o parecer número 1.830 .887$.

A pesquisa foi desenvolvida no Centro Saúde Escola do Marco (CSE-MARCO), localizado em Belém-PA, no período de janeiro a abril de 2017 . 
Foram incluídas na amostra 199 puérperas com idade entre 18 e 40 anos, e que estivessem no puerpério no período máximo de um ano. Foram excluídas do estudo mulheres analfabetas, pois o questionário é autoaplicável.

As participantes foram abordadas no CSE-MARCO quando aguardavam por suas consultas e convidadas a participar da pesquisa, e então direcionadas para uma sala reservada, onde assinavam o termo de consentimento livre e esclarecido e em seguida respondiam ao questionário de Experiência e Satisfação com o Parto ${ }^{14}$ modificado.

Esse questionário é autoaplicável, constituído por um total de 104 questões referentes às expectativas, experiência, satisfação e dor relativas ao trabalho de parto, parto e pós-parto imediato. As perguntas relativas (em vez de: respeitantes) à experiência, satisfação e dor são do tipo Lickert, numa escala que varia entre 1 e 4 ("nada", "um pouco", "bastante", "muito"). As questões que se reportam às expectativas também são do tipo Lickert, numa escala que varia entre 1 e 4 ("muito pior", "pior", "melhor", "muito melhor" ou "muito menos", "menos", "mais", "muito mais"). As questões que se relacionam com a intensidade da dor, embora sejam igualmente do tipo Lickert, variam numa escala entre 0 e 10 ("nenhuma", "mínima”, "muito pouca", "pouca”, "alguma”, "moderada”, "bastante”, "muita”, “muitíssima”, “extrema”, “a pior jamais imaginável”).$^{13}$ Foram acrescentadas perguntas, pelos pesquisadores, que abordavam o conhecimento das puérperas sobre os MAP, se durante a gravidez elas receberam alguma orientação para exercitá-lo ou como prepará-lo para o parto vaginal.
Os dados foram analisados por meio de estatística descritiva, buscando-se a frequência de respostas em cada pergunta.

\section{Resultados}

O estudo foi realizado com 199 puérpe-

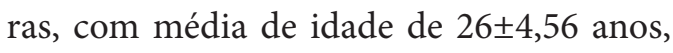
mínimo de 18 e 35 anos. A frequência de distribuição dos dados sobre história obstétrica está descrita na Tabela 1. Observa-se que a frequência de partos vaginais é maior do que de partos cesáreos.

Tabela 1 - Distribuição dos dados sobre a história obstétrica $(n=199)$.

\begin{tabular}{c|c|c|}
\hline Variáveis & $\mathbf{N}$ & $\mathbf{\%}$ \\
\hline $\begin{array}{c}\text { Quantidade de partos } \\
\text { vaginais }\end{array}$ & & \\
0 & 69 & 34,67 \\
1 & 95 & 47,74 \\
2 & 31 & 15,58 \\
3 & 2 & 1,01 \\
5 & 1 & 0,50 \\
6 & 1 & 0,50
\end{tabular}

Quantidade de partos

cesáreos

$\begin{array}{lll}0 & 83 & 41,71 \\ 1 & 94 & 47,24 \\ 2 & 22 & 11,06\end{array}$

Quantidade de abortos

\begin{tabular}{ccc}
0 & 166 & 83,42 \\
1 & 30 & 15,08 \\
2 & 3 & 1,51 \\
\hline
\end{tabular}

A respeito da satisfação das puérperas sobre o seu atendimento no pré-natal, descrito na Tabela 2 , a maioria $(53,27 \%)$ relata que o atendimento foi bom e estar satisfeita com 
o pré-natal. A maior parte refere também que desejava ter parto vaginal e que esse tipo de parto foi o mais incentivado no pré-natal.

Tabela 2 - Frequência das respostas em relação à satisfação do atendimento no pré-natal ( $\mathrm{n}=199)$.

\begin{tabular}{|c|c|}
\hline Perguntas & $\%$ \\
\hline \multicolumn{2}{|l|}{$\begin{array}{l}\text { O que você achou do seu } \\
\text { atendimento pré-natal? }\end{array}$} \\
\hline Ótimo & $27,64 \%$ \\
\hline Muito bom & $5,03 \%$ \\
\hline Bom & $53,27 \%$ \\
\hline Ruim & $12,06 \%$ \\
\hline Péssimo & $2,01 \%$ \\
\hline \multicolumn{2}{|l|}{$\begin{array}{l}\text { Como você se sente em relação à } \\
\text { assistência pré-natal que recebeu? }\end{array}$} \\
\hline Satisfeita & $73,37 \%$ \\
\hline Insatisfeita & $11,56 \%$ \\
\hline Nem satisfeita, nem insatisfeita & $15,08 \%$ \\
\hline \multicolumn{2}{|l|}{ O seu parto foi o que você desejava? } \\
\hline Sim & $65,83 \%$ \\
\hline Não & $34,17 \%$ \\
\hline \multicolumn{2}{|l|}{ Qual tipo de parto você desejava? } \\
\hline Vaginal & $68,84 \%$ \\
\hline Cesáreo & $31,16 \%$ \\
\hline \multicolumn{2}{|l|}{$\begin{array}{l}\text { Você foi incentivada a ter que tipo de } \\
\text { parto? }\end{array}$} \\
\hline Vaginal & $46,23 \%$ \\
\hline Cesáreo & $43,72 \%$ \\
\hline Nenhum & $10,05 \%$ \\
\hline
\end{tabular}

Na Tabela 3 está descrita a frequência de orientações recebidas em relação à preparação do assoalho pélvico. Observa-se que $60,80 \%$ das mulheres não têm conhecimento do assoalho pélvico e não recebem orientação para trabalhar essa musculatura, apesar de o assoalho pélvico ser uma mus- culatura importante que vai ser muito exigida na gravidez e parto.

Tabela 3 - Frequência de respostas acerca do assoalho pélvico.

\begin{tabular}{|c|c|}
\hline Perguntas & $\%$ \\
\hline \multicolumn{2}{|c|}{ Você sabe o que é assoalho pélvico? } \\
\hline Sim & 39,20 \\
\hline Não & 60,80 \\
\hline
\end{tabular}

Você foi orientada por algum profissional da área da saúde para preparar os MAP para ter um parto vaginal?

\begin{tabular}{cc} 
Sim & $7,04 \mid$ \\
Não & 92,96 \\
\hline
\end{tabular}

Em relação à presença de disfunções do assoalho pélvico, descritas na Tabela 4, observa-se que $38,69 \%$ relataram perder urina involuntariamente e apenas a minoria, $14,57 \%$, das mulheres apresentou disfunção fecal.

Tabela 4 - Frequência das respostas em relação à presença de disfunções do assoalho pélvico.

\begin{tabular}{cc}
\hline \multicolumn{1}{c}{ Perguntas } & $\%$ \\
\hline Você está com perda de urina? & \\
Sim & 38,69 \\
Não & 61,31 \\
Você está com perda de fezes? & \\
Sim & 14,57 \\
Não & 85,43 \\
\hline
\end{tabular}

\section{DIscussão}

O presente estudo verificou o nível de orientação oferecido às mulheres durante $o$ seu pré-natal a respeito do preparo dos MAP e a sua satisfação quanto ao pré-natal recebido. 
Nesta pesquisa, observou-se uma maior prevalência de partos normais em relação aos partos cesáreos, o que é comum e incentivado em maternidades públicas, seguindo as recomendações da Organização Mundial da Saúde, que orienta que a taxa de cesariana não deve ultrapassar os $15 \%$ na rede pública. ${ }^{15}$

Quase metade das participantes deste estudo relatou que elas foram incentivadas pela equipe de pré-natal a terem o parto vaginal, assim também, o desejo da maioria delas era o parto vaginal. Apesar desses dados, a forma de assistência dada à mulher influencia no tipo de parto, de maneira que as mulheres do setor privado são levadas a decidir por uma cesariana e as do setor público, mesmo preferindo o parto vaginal, não são apoiadas na sua opção até o final da gestação. ${ }^{16}$ A preferência pelo parto vaginal decorre de uma recuperação mais rápida e mais facilidade para cuidar do filho. ${ }^{17}$ Entre as razões para o parto cesáreo estão a preferência médica e o medo da dor do parto vaginal pelas mulheres, ${ }^{17}$ que é agravado por uma assistência malconduzida de trabalho de parto, tornando o parto vaginal uma experiência dolorosa. ${ }^{16,18}$

Portanto, a atuação multidisciplinar dos profissionais de saúde durante o pré-natal é fundamental para esclarecer as gestantes e familiares sobre os riscos e benefícios dos diferentes tipos de partos, o que conduz à diminuição da ansiedade e garante um sentimento de segurança e confiança às gestantes. ${ }^{18}$ As participantes deste estudo se mostraram satisfeitas, em sua maioria, com o pré-natal oferecido.

Outro ponto importante a ser observado é que a maioria das participantes não teve orientação para preparar os MAP para o parto vaginal, do mesmo modo a maior parte nem mesmo sabia da existência dos mesmos. Os MAP são importantes para sustentação dos órgãos pélvicos, manutenção da continência urinária e fecal e para a função sexual. ${ }^{19}$ Contudo, durante a gestação os MAP sofrem alterações que afetam o seu tônus muscular e força muscular, podendo ocasionar lesões. ${ }^{6}$ Tais alterações, na grande maioria, são decorrentes das alterações hormonais e mudanças anatômicas, ${ }^{20}$ nas quais se destacam o aumento do volume uterino ocasionando a distensão progressiva do tecido conjuntivo dos MAP, além da sobrecarga excessiva pelo aumento da massa corporal materna. ${ }^{6}$

A maioria das participantes deste estudo não relatou apresentar DAP. Deve-se ressaltar que, embora o impacto do parto no desenvolvimento das DAP seja claro, outros fatores incitantes e intervenientes desempenham um papel crítico, como a obesidade, que deve ser combatida. Ademais, nem todas as mulheres com fatores de risco obstétrico desenvolvem DAP sintomáticas e algumas permanecem assintomáticas ou experimentam sintomas transitórios, e outras desenvolvem sintomas significativos muito mais tarde na vida. ${ }^{21}$

Nesse sentido, Varela et al. ${ }^{22}$ observaram em seu estudo que a paridade, o tipo de parto e o nível de atividade física parecem não ter influência na função dos MAP em mulheres pós-menopáusicas, de forma que o avanço da idade seria o responsável pelo declínio na função dos MAP, e que isso é comum a todas as mulheres, independentemente das condições obstétricas anteriores.

Colla et al. ${ }^{23}$ compararam o assoalho pélvico de 227 puérperas de parto vaginal, 
parto cesáreo eletivo e parto cesáreo não eletivo e concluíram que o tipo de parto não foi associado a diferenças no desenvolvimento a curto prazo das DAP pós-parto.

No entanto, Blomquist et al. ${ }^{24}$ descreveram a incidência das DAP em 1.528 mulheres, após uma e duas décadas pós-parto, observando que o parto cesáreo foi associado a um risco significativamente menor de IU de esforço, bexiga hiperativa e prolapso de órgãos pélvicos, em relação ao parto vaginal espontâneo, enquanto o parto vaginal operatório foi associado a um risco significativamente maior de incontinência anal e prolapso de órgãos pélvicos. O risco de desenvolver incontinência anal pós-parto cesáreo também foi percebido por Larsson et al., ${ }^{25}$ mas esse risco seria maior se o parto fosse vaginal.

Em uma revisão sistemática com metanálise, Yang e Sun ${ }^{26}$ descreveram que o parto cesáreo é o modo de parto preferido para as gestantes em termos de função do assoalho pélvico. Porém, a indicação de parto cesáreo deve levar em consideração outros riscos para a saúde da mãe e do feto como complicações cirúrgicas, cardiovasculares e infecções etc. ${ }^{25-27}$ Por isso, a cesariana deve ser discutida com a paciente apenas se houver um risco aumentado de perda funcional do trabalho de parto, e não apenas para os propósitos de evitar danos no assoalho pélvico e possível comprometimento da IU pós-parto. ${ }^{27}$

No entanto, mulheres que não exercitam o assoalho pélvico, durante a gravidez, estão mais propensas a desenvolver algum tipo de DAP, principalmente a IU. ${ }^{18}$ Entretanto, as DAP são passiveis de prevenção e tratamento. Quando o treinamento dos
MAP (TMAP) é aplicado durante a gravidez, os benefícios são diversos, como o aumento da força dos MAP, prevenindo a IU ${ }^{5}$, aumento das chances de partos vaginais, redução da ansiedade, depressão, redução do quadro álgico, melhora da qualidade de vida, melhora na satisfação pessoal e na percepção da saúde materna. ${ }^{28} \mathrm{~A}$ reabilitação do assoalho pélvico é prescrita como tratamento de primeira linha para mulheres com IU e pode realmente ser uma medida de proteção viável contra a IU durante o período pós-parto. ${ }^{27}$

Nesse seguimento, Leon-Larios ${ }^{29}$ observou que o TMAP e massagem perineal para mulheres primíparas aumentam a probabilidade de um períneo íntegro, reduz as taxas de episiotomia e trauma perineal grave, dor perineal pós-parto e não possuemqualquer influência nos resultados perinatais no recém-nascido. Assim também, Pereira et al., ${ }^{29}$ em uma revisão sistemática, demonstraram que o uso do Epi-No durante o pré-natal é eficaz na redução de laceração e episiotomia pósparto, de forma segura para a mãe e filho e de fácil utilização.

Todavia, orientações específicas durante o pré-natal e puerpério quanto aos cuidados com o MAP não são fornecidas na rotina dos programas do Ministério da Saúde. ${ }^{6}$ Tais orientações são importantes para ajudar na decisão da mulher quanto ao tipo de parto e prevenir DAP durante a gestação e no período pós-parto. ${ }^{6}$

Assim, a orientação acerca da função dos MAP deveria ser dada em vários momentos da vida, ${ }^{31}$ principalmente para mulheres expostas aos fatores de risco de DAP como a gravidez e o parto, fazendo, portanto, necessário que as equipes de profissio- 
nais de saúde envolvidas com a atenção pré-natal, incluindo o fisioterapeuta, possam se capacitar quanto a esses cuidados. ${ }^{6}$

Sugerem-se que mais estudos de ensaios clínicos controlados sejam realizados com a finalidade de preparar os MAP durante o pré-natal para o parto e os resultados possam ser avaliados em longo prazo, além de estudos que avaliem somente os efeitos da gravidez e parto nos MAP, eliminando os demais fatores de risco para DAP.

\section{CONSIDERAÇões FINAIS}

O estudo permitiu concluir que a maioria das mulheres não recebeu orien- tação para preparar os MAP para o parto durante o pré-natal e apenas a minoria tinha conhecimento sobre o que é o assoalho pélvico.

Apesar de os MAP serem um grupo muscular bastante solicitado durante a gravidez e parto, o TMAP ou mesmo a orientação para exercitar os MAP ainda não é rotina nos serviços públicos.

É necessário que durante a consulta de pré-natal a gestante receba orientações a respeito da importância da preparação dos MAP, a fim de prevenir futuras DAP, e proporcionar uma melhor qualidade de vida à gestante e ao bebê durante o trabalho de parto e pós-parto.

\section{REFERÊNCIAS}

1. Rodrigues EM, Nascimento RG, Araújo A. Protocolo na assistência pré-natal: ações, facilidades e dificuldades dos enfermeiros da Estratégia de Saúde da Família: Rev Esc Enferm USP, São Paulo, 2011; 45 (5): 1.041-1.047.

2. Soares PRAL. Disfunção do assoalho pélvico e qualidade de vida relacionada à saúde de gestantes. 2015. 163 f. Tese (Doutorado) - Faculdade de Farmácia, Odontologia e Enfermagem, Universidade Federal do Ceará, Fortaleza, 2015.

3. Ferederice CP, Amaral E, Ferreira NO. Sintomas urinários e função muscular do assoalho pélvico após o parto. Rev Bras Ginecol Obstet. 2011; 33 (4): 188-95.

4. Pinheiro BF, Franco GR, Feitosa SM, Yuaso DR, Castro RA, Girão MJBC. Fisioterapia para consciência perineal: uma comparação entre as cinesioterapias com toque digital e com auxílio do biofeedback, 2012; 21 (1): 639-648.

5. Mendes EPB, Oliveira SMJV, Caroci AS, Francisco AA, Oliveira SG, Silva RL. Força muscular do assoalho pélvico em primíparas segundo o tipo de parto: estudo transversal. Rev Latino-Am. Enfermagem. 2016; 24: e 2.758.

6. Moccellin AS, Rett MT, Driusso P. Existe alteração na função dos músculos do assoalho pélvico e abdominais de primigestas no segundo e terceiro trimestre gestacional. Fisioter Pesqui. 2016; 23 (2): 136-41.

7. Luber KM, Boero S, Choe JY. The demographics of pelvic floor disorders: current observations and future projections. Am J Obstet Gynecol. 2001;184(7):1496-501

8. Santos PC, Mendonça D, Alves O, Barbosa AM. Prevalência e impacto da incontinência urinária de estresse: antes e durante a gravidez. Acta Med Port. 2006;19:349-56.

9. Marques LP, Schneider IJC, Giehl MWC, Antes DL, D’Orsi E. Demographic, health conditions, and lifestyle factors associated with urinary incontinence in elderly from Florianópolis, Santa Catarina, Brazil. Rev Bras Epidemiol. 2015;18(3):595-606. 
10. Kessler M., Facchini LA, Soares MU, Nunes BP, França SM, Thumé E. Prevalência de incontinência urinária em idosos e relação com indicadores de saúde física e mental. Rev. bras. geriatr. gerontol. 2018; 21 (4): 397-407.

11. Alves JO, Da Luz ST, Brandão S., Da Luz CM, Jorge RN, Da Roza T. Urinary Incontinence in Physically Active Young Women: Prevalence and Related Factors. Int J Sports Med. 2017; 38 (12): 937-941.

12. Carvalho MP, Andrade FP, Peres W, Martinelli T, Smich F, Orcy RB, Seleme MR. O impacto da incontinência urinaria e seus fatores associados em idosas. Rev. Bras. Geriatr. Gerontol. 2014; 17 (4): 721-730.

13. Parente LC, Henriques LHF da C. Fisioterapia na incontinência urinária no pós-parto. Fisioterapia Brasil. 2007;8(4):288-293

14. Costa R, Figueiredo B, Pacheco A, Marques A, Pais A. Questionário de experiência e satisfação com o parto (QESP). Psicologia, saúde \& doenças. 2004; 12 (3): 159-187.

15. Organização mundial de saúde. Maternidade segura: atenção ao nascimento normal: um guia prático. Genebra: Organização mundial de saúde, 1996.

16. Domingues RMSM, Dias MAB, Pereira MN, Torres JA, D’orsi E, Pereira APE, Schilithz AOC, et al. Processo de decisão pelo tipo de parto no Brasil: da preferência inicial das mulheres à via de parto final. Cad. Saúde Pública, Rio de Janeiro, 2014; 30: 101-116.

17. Lamy GO, Moreno BS. Assistência pré-natal e preparo para o parto. Omnia Saúde. 2013; 10: 19-35.

18. Santos RAA, Melo MCP, Leal RJM. Experiência do tipo de parto: relato de puérperas através da análise de discurso. Rev Ciênc Méd Biol, Salvador, 2015; 74-81.

19. Assis LC. Evolução da função muscular do assoalho pélvico no pós-Parto. 2013. $91 \mathrm{f}$. Tese (doutorado) - Universidade Estadual Paulista Julio de Mesquita Filho, Faculdade de Medicina de Botucatu, 2013.

20. Batista RLA, Franco MM, Naldoni LMV, Duarte G, Oliveira AS, Ferreira CHJ. Biofeedback na atividade eletromiográfica dos músculos do assoalho pélvico em gestantes. Rev Bras Fisioter, São Carlos, 2011; 15 (5): 386-92.

21. Halock JL, Honda VL, The epidemiology of pelvic floor disorders and childbirth: an update. Obstet Gynecol Clin North Am. 2016; 43 (1): 1-13.

22. Varella LRD, Torres VB, Angelo PHM, Oliveira MCE, Barros ACM, Viana ESR, Micussi MTABC. Influence of parity, type of delivery, and physical activity level on pelvic floor muscles in postmenopausal women. J. Phys Ther Sci. 2016; 28: 824-830.

23. Colla C., Paiva LL, Ferla L., Trento MJB, De Vargas IMP, Dos Santos BA, et al. Pelvic floor dysfunction in the immediate puerperium, and 1 month and 3 months after vaginal or cesarean delivery. Int J Gynaecol Obstet. 2018; 143 (1): 94-100.

24. Blomquist JL, Munoz A., Carrol M., Handa VL. Association of Delivery Mode With Pelvic Floor Disorders After Childbirth. JAMA. 2018. Dec 18; 320 (23): 2.438-2.447.

25. Larsson C., Hedberg $\underline{\text { CL}}$, Lundgren $\underline{E}$., L. Söderström, Lars BS, TunÓn K., Nordin P. Anal incontinence after caesarean and vaginal delivery in Sweden: a national population-based study. The lancet. 201930 (13): 1.552-1.555.

26. Yang XJ, Sun Y. Comparison of Caesarean Section and Vaginal Delivery for Pelvic Floor Function of Parturients: A Meta-Analysis. Eur J Obstet Gynecol Reprod Biol. 2019; 235: 42-48.

27. Wang H., Ghoniem G. Postpartum stress urinary incontinence, is it related to vaginal delivery? J Matern Fetal Neonatal Med. 2017; 30 (13): 1.552-1.555.

28. Riscado LC, Jannotti CB, Barbosa RHS. A decisão pela via de parto no Brasil: temas e tendências na produção da saúde coletiva. Enferm, Rio de Janeiro, 2016; 25 (1): e 3570014. 
29. Larilos FL, Gutierrez IC, Mejía RC, Serrano CS. Influence of a pelvic floor training programme to prevent perineal trauma: a quase-randomised controlled Trial. Midwifery. 2017; 50: 72-77.

30. Pereira AG, Oliveira AMB, Cury GD, Jorge LB, Latorre GS. Eficácia do Epi-No na diminuição da episiotomia e risco de lesão perineal pós-parto. Revisão sistemática, Curitiba, 2015.

31. Neels H, Wyndaele JJ, Wiebren AAT, Wachter S, Wyndaele M, Vermandel A. Knowledge of the pelvic floor in nulliparous women. J. Phys. Ther. Sci. 2016; 28: 1.524-1.533.

\section{DAdos DOS AUTORES}

\section{ANdressa CÂmara Rodrigues}

Discente do curso de Fisioterapia da Universidade da Amazônia. Atualmente trabalha na Clínica Cafisio Mulher. Belém/PA - Brasil. andressacrcr@gmail.com

\section{VANESSA ÂNGELA DE OLIVEIRA}

Discente do curso de Fisioterapia da Universidade da Amazônia. Belém/PA - Brasil. vanessa_oliiveira11@yahoo.com.bb

\section{SAÚL RASSY CARNEIRO}

Doutor em Doenças Tropicais. Fisioterapeuta do Hospital Universitário João de Barros Barreto e da Secretaria Especial Saúde Pública do Estado do Pará. Belém/PA - Brasil. saul_rassy@yahoo.com.br

\section{Cibele Nazaré Câmara Rodrigues}

Doutora em psicologia. Professora do curso de fisioterapia da Universidade Federal do Pará. Belém/ PA - Brasil. cibelecamara@hotmail.com

\section{DAYSE DANIELLE DE O. SILVA}

Doutora em Ciências da Reabilitação. Professora do Curso de Fisioterapia da Universidade do Estado do Pará. Belém/PA - Brasil. daysesilva@uepa.br

\section{Gustavo F. Sutter LAtorre.}

Mestre em fisioterapia. Fisioterapeuta pélvico. Florianópolis/SC - Brasil. gustavo@perineo.net

\section{Erica Feio Carneiro Nunes}

Doutora em Ciências da Reabilitação. Professora do Curso de Fisioterapia da Universidade do Estado do Pará. Belém//PA - Brasil. erica@perineo.net

Submetido em: 28-5-2019

Aceito em: 15-8-2020 